\title{
Efetividade de um coxim de cabina do trator agrícola na atenuação das vibrações ${ }^{1}$
}

\author{
Effectiveness of a tractor cab cushion on the mitigation of vibrations
}

\author{
Marivan da Silva Pinho²*, José Fernando Schlosser³, Ulisses Giacomini Frantz ${ }^{2}$, Fabricio Azevedo Rodrigues² e \\ Pablo Silva Ferrer ${ }^{2}$
}

\begin{abstract}
RESUMO - A vibração propagada através do trator interfere diretamente no operador, desta forma, para minimizar os problemas é desejável analisar os pontos críticos desta propagação. O operador do trator está exposto aos efeitos nocivos da vibração na sua saúde, além de diminuir sua capacidade de trabalho por efeito da fadiga, sendo oportunas maiores investigações dos parâmetros de frequência da vibração nos coxins. Este trabalho teve por objetivo analisar a transmissibilidade das vibrações no coxim elastomérico de um trator agrícola com cabine utilizando duas profundidades de escarificação (15 e $30 \mathrm{~cm}$ ), duas velocidades de deslocamento $\left(3,5\right.$ e 4,5 $\left.\mathrm{km} \mathrm{h}^{-1}\right)$ e três configurações de lastros (metálico total, metálico parcial e líquido total) com relações de peso/potência de 49,5; 43 e 49 kg kW-1 respectivamente. O experimento foi realizado na Universidade Federal de Santa Maria com um delineamento de parcelas subdivididas. Utilizou-se um trator, com potência no motor de $62,5 \mathrm{~kW}(85 \mathrm{cv})$, tendo um escarificador da marca Jan acoplado, os dados foram registrados através de acelerômetros (Type $4513 \mathrm{~B}$ marca Bruel \& Kjaer) instalados no piso da cabina, na plataforma e no coxim. Concluiu-se que a transmissibilidade de vibração na direção vertical através do coxim teve amplificação no intervalo de 0 a $15 \mathrm{~Hz}$ para a Lastro metálico total, de 0 a $19 \mathrm{~Hz}$ para a Lastro metálico parcial e de 71 a $76 \mathrm{~Hz}$ para a Lastro líquido total.
\end{abstract}

Palavras-chave: Tratores agrícolas. Vibração. Ergonomia.

\begin{abstract}
Vibration propagated through the tractor directly affects the operator, therefore to minimise problems, it is desirable to analyse the critical points of this propagation. The tractor operator is exposed to the harmful effects of the vibration on their health, besides experiencing a reduction in their ability to work as a result of fatigue. More investigation into the parameters of vibration frequencies in the cushions therefore becomes necessary. The objective of this study was to analyse the transmissibility of vibrations in the elastomeric cushion of an agricultural tractor with cab, using two depths of scarification (15 and $30 \mathrm{~cm}$ ), at two speeds ( 3.5 and $4.5 \mathrm{~km} \mathrm{~h}^{-1}$ ) and three ballast configurations (all metal, part metal and all liquid), with weight to power ratios of $49.5,43$ and $49 \mathrm{~kg} \mathrm{~kW}^{-1}$ respectively. The experiment was carried out at the Federal University of Santa Maria, in the State of Rio Grande do Sul, Brazil, using a split-lot design. A tractor with an engine power of $62.5 \mathrm{~kW}(85 \mathrm{cv})$ coupled to a Jan-branded chisel plough was used, with the data being recorded using accelerometers (Type 4513 B marca Bruel \& Kjaer) installed on the cab floor, on the platform and on the cushion. It was concluded that vibration transmissibility through the cushion in the vertical direction was amplified in the range of $0-15 \mathrm{~Hz}$ for all-metal ballast, $0-19 \mathrm{~Hz}$ for part-metal ballast, and 71-76 Hz for all-liquid ballast.
\end{abstract}

Key words: Agricultural tractors. Vibration. Ergonomics.

\footnotetext{
*Autor para correspondência

${ }^{1}$ Recebido para publicação em 07/11/2012; aprovado em 05/03/2014

Parte da Tese de Doutorado do primeiro autor apresentada ao Curso de Pós-Graduação em Engenharia Agrícola/UFSM, Santa Maria-RS

${ }^{2}$ Programa de Pós-Graduação em Engenharia Agrícola/PPGEA, Universidade Federal de Santa Maria, Santa Maria-RS, Brasil, marivanpinho@ hotmail.com, ulissesgf@hotmail.com, fabricioazevedorodrigues@hotmail.com, psilvaferrer@gmail.com

${ }^{3}$ Bolsista de produtividade CNPq, Departamento de Engenharia Rural, Centro de Ciências Rurais, Universidade Federal de Santa Maria, Santa

Maria-RS, Brasil, josefernandoschlosser@gmail.com
} 


\section{INTRODUÇÃO}

A jornada de trabalho do operador no trator agrícola é bastante árdua devido o mesmo estar exposto às vibrações (SCHLOSSER et al., 2005; SILVA et al., 2011).

Kumar et al. (2001) observaram que as vibrações produzem lesões no abdomem e na coluna vertebral. As pesquisas de vibrações em tratores agrícolas se direcionam aos modelos de pneus e tipos de transmissão (SERVADIO; MARSILI; BELFORE, 2007).

Sam e Kathirvel (2006), observaram que os principais efeitos da vibração no corpo humano reduzem a eficiência na execução das atividades operacionais, estando de acordo com Minette et al. (2007), os quais afirmam que o rendimento do operador é função do aspecto ergonômico do seu posto de trabalho. Verifica-se que as inovações em projetos de tratores, com conceitos ergonômicos promovem os melhores resultados para o operador. Segundo Yulan (2005), variáveis como: espessura, tipo de material e design são soluções para problemas ergonômicos do operador. Rychtyckyj (2005), relata que a utilização do software Modapts permite a criação de modelos virtuais que geram funções de conforto para o corpo humano.

Um dos segmentos de estudos da ergonomia corresponde às vibrações ocupacionais, que são amplamente reconhecidas como um fator de risco para os trabalhadores. Nietiedt et al. (2012), relatam que a análise ergonômica do posto de operação de tratores agrícolas contribui para melhor proteção e conforto ao operador.

Os coxins são isoladores elastoméricos, que minimizam a magnitude de vibração produzida do motor para a cabina, sendo a faixa de 0 a $200 \mathrm{~Hz}$ o intervalo que permite a caracterização completa da transmissibilidade através do coxim. A transmissibilidade através do coxim é a relação estabelecida entre a razão da força transmitida na parte superior do coxim e a força excitante na parte inferior do coxim ou a razão da magnitude transmitida e a magnitude excitante (BARBETTI, 2005; IIDA, 2000).

A principal direção a ser analisada, tendo em vista o conforto do operador, é a direção vertical (FRANCHINI, 2007). Desta forma, segundo Oliveira (2011) e Franchini (2007) são condizentes pesquisas que avaliem as frequências por onde ocorre à transmissibilidade nesta direção, a fim de aperfeiçoar os elementos de absorção.

Barbetti (2005), relata que para reduzir a transmissibilidade de vibração é fundamental que a frequência natural do coxim seja inferior a frequência originada do motor, ou seja, é necessária baixa rigidez (maciez) para isolar altos valores de frequência.

Os pontos de apoio do motor em relação à estrutura são posicionados de forma que a carga do motor seja distribuída proporcionalmente aos coxins. A isolação através do coxim serve para amenizar os efeitos da combustão durante a explosão e o movimento dos componentes mecânicos tais como: manivela e virabrequim (BARBETTI, 2005).

Freitas (2006) recomenda que o coxim ideal deve apresentar alta rigidez e alto amortecimento para reduzir a transmissibilidade em frequências inferiores a $30 \mathrm{~Hz}$, baixa rigidez e baixo amortecimento para reduzir as frequências de 30 a $200 \mathrm{~Hz}$ que correspondem à faixa de frequência produzida pelas rotações do motor.

Desta forma este trabalho tem por objetivo caracterizar as faixas de frequências nas quais ocorre a amplificação da transmissibilidade através do coxim no espectro de 0 a $200 \mathrm{~Hz}$ e determinar os valores médios da transmissibilidade no espectro de $1 \mathrm{a} 80 \mathrm{~Hz}$.

\section{MATERIAL E MÉTODOS}

$\mathrm{O}$ experimento foi realizado durante os meses de outubro e novembro de 2011, no campo experimental do Departamento de Fitotecnia da Universidade Federal de Santa Maria, Rio Grande do Sul, Brasil. As coordenadas geodésicas do ponto médio são: $29^{\circ} 43^{\prime} 32,30^{\prime \prime} \mathrm{S}$ e $53^{\circ} 43^{\prime} 14,62^{\prime \prime} \mathrm{O}$. O local onde foi realizado o experimento tem característica de um campo nativo com solo rugoso, classificado como Argissolo Vermelho distrófico arênico (EMPRESA BRASILEIRA DE PESQUISA AGROPECUÁRIA, 1999).

$\mathrm{O}$ trator utilizado foi da marca Massey Ferguson, modelo MF4283 com cabine, 4 × 2 TDA, ano 2011, potência do motor de $62,5 \mathrm{~kW}(85 \mathrm{cv}), 4$ cilindros e volume de $4.100 \mathrm{~cm}^{3}$, rotação utilizada de $2.200 \mathrm{rpm}$. Os rodados utilizados no trator foram, traseiros R1 18.4-30 de 10 lonas, e dianteiros R1 12.4-24 de 6 lonas TM 95. Utilizou-se o escarificador da marca Jan, montado: acoplado ao Sistema Hidráulico de Três Pontos (SHTP) do trator. O escarificador tem a seguinte especificação: 3 hastes em forma de curva parabólica, ponteira estreita de $0,08 \mathrm{~m}$ sem asa, ângulo de ataque de $20^{\circ}$ (ângulo formado entre a ponteira e o solo) e rodas limitadoras de profundidade acopladas ao chassi.

Foram utilizados três fatores com distintos níveis ou tratamentos no experimento: relação massa/potência e tipo de lastro; velocidade de deslocamento do trator 
e profundidade de escarificação. Abaixo são descritos os níveis de cada um destes fatores e a metodologia utilizada para a sua obtenção. Os tratamentos de Lastro Metálico Total (LMT) e Lastro Metálico Parcial (LMP) foram obtidos através da adição de pesos metálicos nos rodados traseiros e no suporte frontal. Na obtenção do Lastro Líquido Total (LLT) foi adicionada água a $75 \%$ do volume interno dos pneus. Na Tabela 1 são verificados os tratamentos e suas relações.

Para a obtenção das velocidades de deslocamento trator-escarificador foi determinado o tempo, através de um cronômetro digital, correspondente ao deslocamento de $100 \mathrm{~m}$, sendo obtidos os níveis de 3,5 e $4,5 \mathrm{~km} \mathrm{~h}^{-1}$, proporcionados pelas marchas $2^{\mathrm{a}}$ reduzida baixa e $2^{\mathrm{a}}$ reduzida alta, respectivamente. Os dois níveis de profundidade de escarificação de 15 e $30 \mathrm{~cm}$, foram obtidos através da alteração da roda limitadora de profundidade.

Realizou-se a combinação do fatorial triplo (2 x 2 x 3) com duas profundidades de escarificação (15 e $30 \mathrm{~cm})$, duas velocidades de deslocamento $(3,5 \mathrm{e}$ $4,5 \mathrm{~km} \mathrm{~h}^{-1}$ ) e três configurações de lastros (metálico total, metálico parcial e líquido total) estabelecendo 12 tratamentos, com 4 repetições cada, totalizando 48 unidades experimentais.

Para cada nível de configurações de lastros, denominado de parcela, foram utilizadas 16 subparcelas, com a casualização dos níveis dos fatores profundidade de escarificação e velocidade de deslocamento. As

Tabela 1 - Tratamentos da relação massa/potência e tipo de lastro

\begin{tabular}{lcccc}
\hline \multirow{2}{*}{ Tratamento } & \multicolumn{2}{c}{ Massa $(\mathrm{kg})$} & MD/MT & \multirow{2}{*}{ MT/P $\left(\mathrm{kg} \mathrm{kW}^{-1}\right)$} \\
\cline { 2 - 5 } & Dianteiro & Traseiro & 0,39 & 67,36 \\
\hline LMT & 1.650 & 2.560 & 0,39 & 58,56 \\
LMP & 1.427 & 2.233 & 0,39 & 66,72 \\
\hline LLT & 1.650 & 2.520 & & 0,36 \\
\hline
\end{tabular}

LMT: lastro metálico total; LMP: lastro metálico parcial; LLT: lastro liquido total; MD: massa transferida ao eixo dianteiro; MT: massa total do trator; P: potência do trator

Figura 1 - Acelerômetros instalados na base inferior e superior do coxim

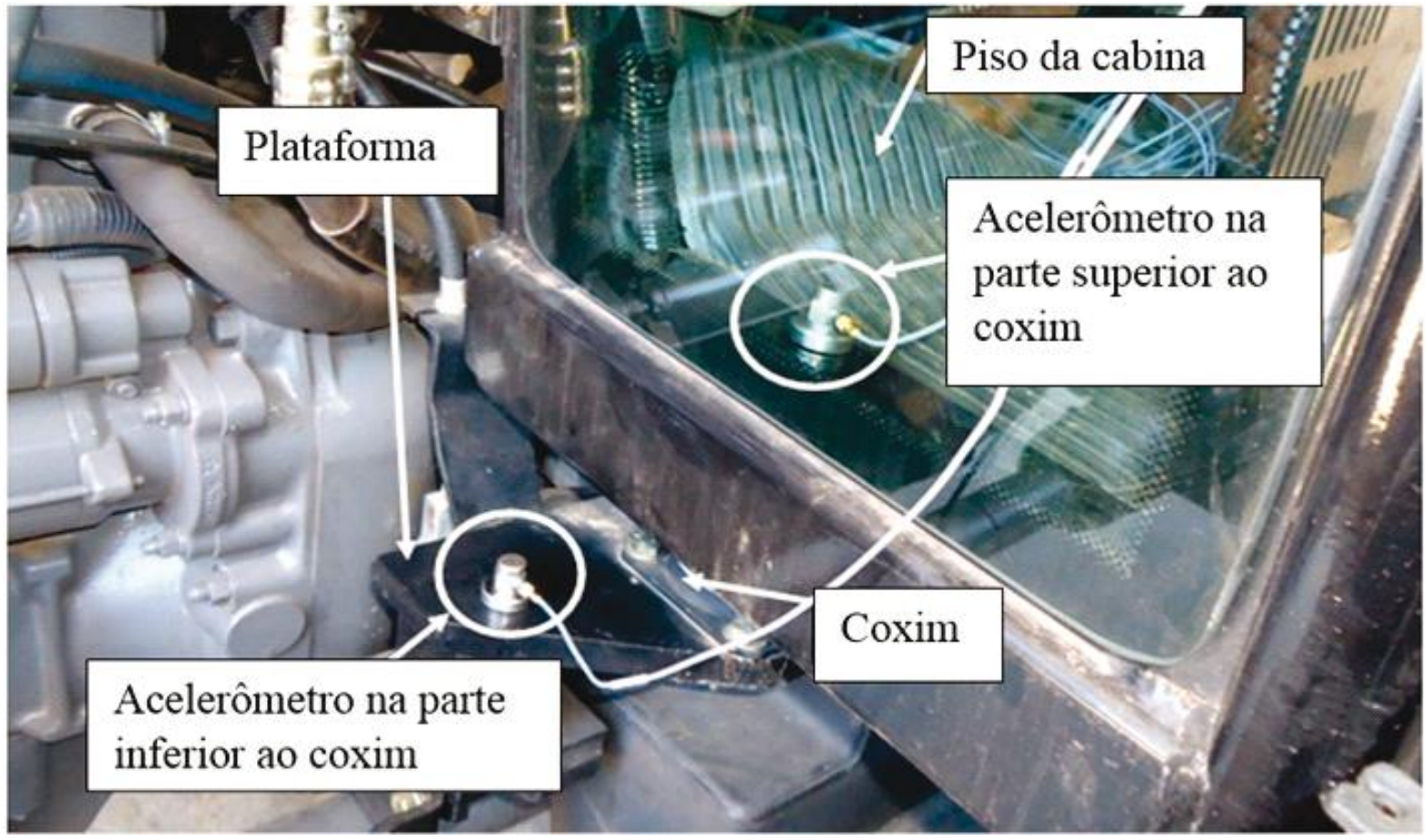


dimensões de cada subparcela foram de $2,5 \mathrm{~m}$ de largura e $100 \mathrm{~m}$ de comprimento, com delineamento de parcelas subdividas.

Para verificar a transmissibilidade através do coxim, a magnitude de vibração no piso da cabina e na plataforma, estrutura metálica de fixação da cabina, foram instalados dois acelerômetros uniaxiais (direção vertical), um na base do coxim, com a especificação Type 4513 B (marca Bruel \& Kjaer) exterior a cabina, e outro na parte superior do coxim (Type 4513 B marca Bruel \& Kjaer) interior a cabina. Os acelerômetros foram colocados sobre uma base metálica e fixados por uma rosca, posteriormente, colocou-se cera na interface da base metálica e na parte metálica da cabina. A transmissibilidade através do coxim permite estabelecer o quanto de vibração é transmitido para a parte interna da cabina (Figura 1).

Os cabos conectados aos acelerômetros uniaxiais foram ligados em dois canais do módulo e fixados na cabina através de fita adesiva. O módulo utilizado foi da Bruel \& Kjaer modelo 3050-B-060 com seis canais que funcionam de maneira independente. A entrada de cada um dos canais tem a tecnologia $D y n-X$, com o reconhecimento inteligente de cada sensor conectado. O módulo foi acoplado à bateria através de um conector e ambos ficaram no suporte lateral da cabina.

Por meio de um cabo Universal Serial Bus (USB) conectou-se o módulo ao Computador portátil. Os dados foram processados simultaneamente através do software Pulse, que é uma plataforma gráfica de análise e simulação de vibração, onde se configurou a faixa de frequência a ser utilizada de 0 a $200 \mathrm{~Hz}$ para a determinação da transmissibilidade através do coxim e da magnitude das acelerações no piso e na plataforma do trator.

Para a obtenção da tensão de $220 \mathrm{~V}$ na parte interna da cabina, em virtude do tempo prolongado para obter a coleta de dados no campo, foi instalado o conversor de tensão que ligado através de cabos na bateria do trator transforma a tensão de $12 \mathrm{~V}$ em $220 \mathrm{~V}$, possibilitando a conexão do conversor ao Computador portátil. Para o tratamento dos dados foi utilizado o software Excel, o qual relaciona o sinal oriundo dos acelerômetros com os limites estabelecidos pela ISO 2631-1: 1997.

Para a determinação da vibração cumulativa foi utilizado o valor dose de vibração (VDV), conforme a equação 1 .

$$
V D V=\left[\frac{T_{s}}{N} \sum x^{4}(i)\right] 1 / 4
$$

Sendo que: VDV é o valor da dose de vibração $\left(\mathrm{m} \mathrm{s}^{-1,75}\right)$; $x$ é a aceleração eficaz ponderada $\left(\mathrm{m} \mathrm{s}^{-2}\right) ; T$ é $o$ período de duração do movimento $(s)$; $\mathrm{N}$ é o número de amostras.

A determinação da transmissibilidade eficaz através do coxim foi determinada conforme a equação 2 .

$$
T=\frac{V D V s}{V D V e}
$$

Sendo que: $T$ é a transmissibilidade (\%); VDVTs é o valor dose de vibração na parte superior $\left(\mathrm{m} \mathrm{s}^{-1,75}\right)$; VDVe é o valor dose de vibração na parte inferior $\left(\mathrm{m} \mathrm{s}^{-1,75}\right)$.

\section{RESULTADOS E DISCUSSÃO}

O uso de lastro metálico total com relação massapotência de $66,72 \mathrm{~kg} \mathrm{~kW}^{-1}$, tem amplificação através do coxim no intervalo de 0 a $15 \mathrm{~Hz}$. Observa-se desta forma, a pouca eficiência de amortecimento do mesmo. De acordo com Oliveira (2011) o coxim elastomérico tem desempenho inferior ao hidráulico.

Na utilização de profundidade de escarificação de $30 \mathrm{~cm}$, não houve amplificação na faixa de 0 a $2 \mathrm{~Hz}$, independente dos níveis de velocidade, no entanto para a profundidade de $15 \mathrm{~cm}$ houve amplificação em todo o intervalo de 0 a $5 \mathrm{~Hz}$. Frequências de 0,1 a $0,7 \mathrm{~Hz}$ propiciam a incidência de enjoos e náuseas (IIDA, 2000). Devido à vibração de baixa frequência ser originada das irregularidades do terreno, na profundidade de $30 \mathrm{~cm}$ o maior esforço de tração não produziu magnitudes satisfatórias para que houvesse amplificação neste intervalo, no entanto, o coxim foi praticamente inerte na absorção.

Verifica-se a baixa eficiência de amortecimento do coxim, visto que de 0 a $15 \mathrm{~Hz}$ houve amplificação, principalmente em um intervalo crítico para o corpo humano, o qual apresenta maior sensibilidade nas frequências de 4 a $8 \mathrm{~Hz}$. No intervalo de 15 a $50 \mathrm{~Hz}$, houve transmissibilidade de mais de $80 \%$, sendo que acima de $50 \mathrm{~Hz}$ o coxim apresentou eficiência satisfatória, transmitindo em torno de $10 \%$ de vibração (Figura 2). Verifica-se a necessidade de se aperfeiçoar os parâmetros ergonômicos (COELHO et al., 2012; GABRIEL FILHO et al., 2010; LLANILLO et al., 2006).

Com lastro metálico parcial ocorre amplificação da transmissibilidade através do coxim no intervalo de 0 a $19 \mathrm{~Hz}$ com exceção do intervalo de 10 a 12 $\mathrm{Hz}$, isso indica que a redução de lastro metálico para uma relação massa/potência de $58,56 \mathrm{~kg} \mathrm{~kW}^{-1}$ não promove amplificação neste intervalo. Comparado com o lastro metálico total, observa-se que uma menor 
Figura 2 - Comportamento da transmissibilidade de vibração através do coxim no intervalo de frequência de 0 a $200 \mathrm{~Hz}$ com lastro metálico total

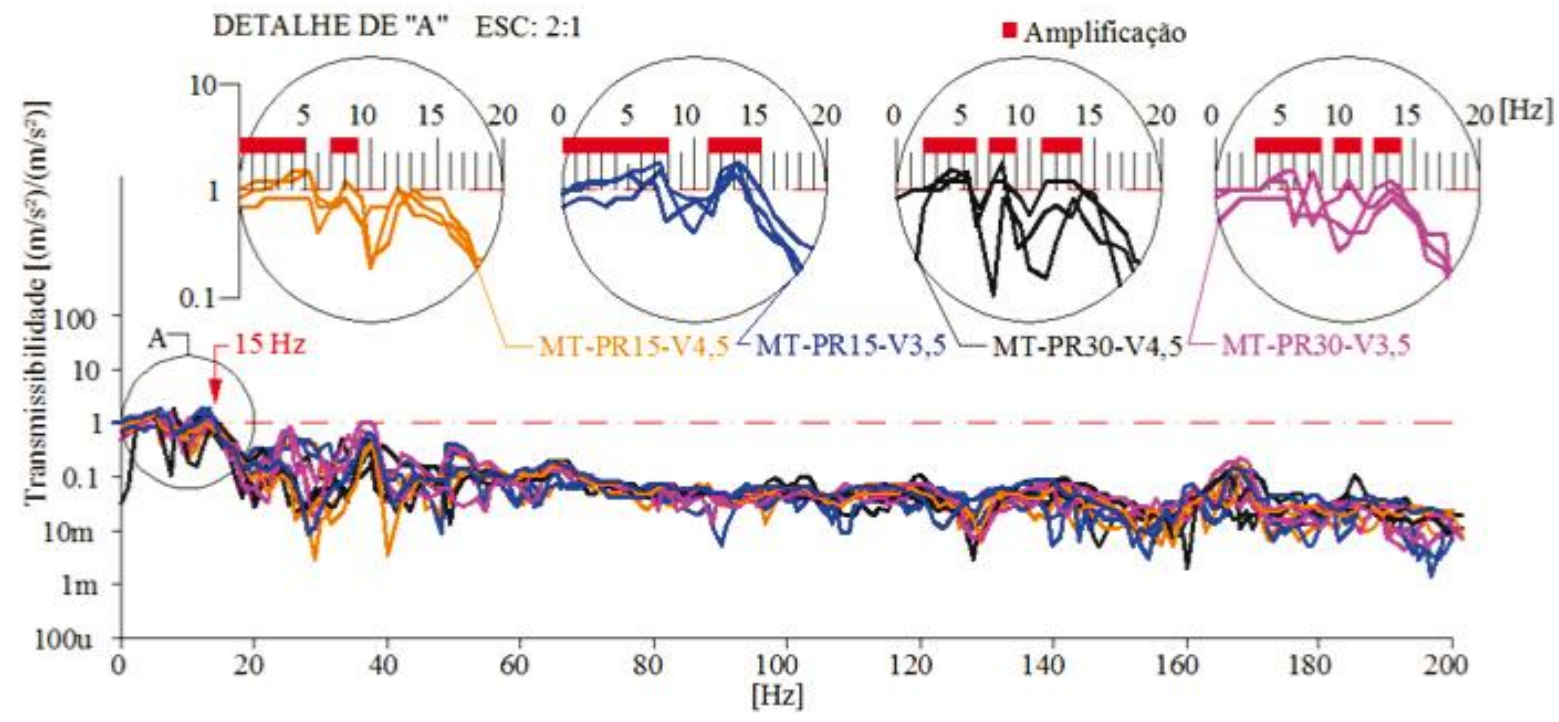

MT- lastro metálico total; PR15- profundidade de escarificação de $15 \mathrm{~cm}$; PR30- profundidade de escarificação de $30 \mathrm{~cm}$; V3,5- velocidade de deslocamento de $3,5 \mathrm{~km} . \mathrm{h}^{-1}$; V4,5- velocidade de deslocamento 4,5 km.h ${ }^{-1}$. Imagem gerada através do software Pulse

relação massa/potência atinge um maior intervalo de frequência amplificado e valores mais elevados na ordem de $200 \%$. Dos 19 aos $50 \mathrm{~Hz}$, o coxim apresenta baixa eficiência de amortecimento, sendo que acima de $50 \mathrm{~Hz}$ a eficiência melhora.

Com velocidade de deslocamento de $3,5 \mathrm{~km} \mathrm{~h}^{-1}$, no intervalo de 7 a $9 \mathrm{~Hz}$, a transmissibilidade excedeu em $150 \%$ os valores na velocidade de 4,5 $\mathrm{km}$. Na velocidade de $3,5 \mathrm{~km} \mathrm{~h}^{-1}$, observa-se maiores picos de transmissibilidade no intervalo de 5 a $10 \mathrm{~Hz}$, evidenciando que a baixa velocidade de deslocamento em condições que impedem o movimento, produz maior transmissibilidade.

$\mathrm{Na}$ baixa relação massa/potência, conservando a lastragem metálica no trator, observa-se uma tendência da amplificação se estender para novas faixas de frequência, uma vez que na relação de $49 \mathrm{~kg} \mathrm{cv}^{-1}$, as faixas atingidas foram de até $15 \mathrm{~Hz}$. No caso de $43 \mathrm{~kg} \mathrm{cv}^{-1}$, as faixas foram até $19 \mathrm{~Hz}$. Como o coxim elastomérico tem rigidez linear não amortece as diferentes condições de operação a campo, para um melhor desempenho de amortecimento, conforme Barbetti (2005) seriam necessárias modificações de parâmetros tais como: geometria, rigidez de amortecimento não linear e material.

Na interface assento-operador, na direção vertical, a maior magnitude foi no intervalo de 2 a $4 \mathrm{~Hz}$ com a operação de gradagem (SANTOS, 2002). Observa-se, assim, que há uma redução da faixa de frequência de amplificação, em relação a operação de escarificação, pois, no coxim, a faixa de variação é de até $19 \mathrm{~Hz}$ e no assento em torno de $2 \mathrm{~Hz}$ (Figura 3).

Com a utilização de lastro líquido total, não se observou transmissibilidade na baixa frequência, no entanto, houve amplificação no intervalo de 71 a $76 \mathrm{~Hz}$, provavelmente esta amplificação seja em função da rotação do motor ser de $2.200 \mathrm{rpm}$ $(36,7 \mathrm{~Hz})$, ou seja, a excitação do motor criou harmônicos de $36,7 \mathrm{~Hz}$, fato este, que propiciou amplificação em torno de 2 vezes $(36,7 \mathrm{~Hz})$. De acordo com IIDA (2000), a vibração no intervalo de 60 a $90 \mathrm{~Hz}$ produz ressonância na pupila dos olhos.

As vibrações de alta frequência são absorvidas em grande parte pelos pneus na interface com o solo, no entanto, a baixa frequência é transmitida ao longo da estrutura do trator através do solo, (BARBOSA et al., 2005). Observam-se valores não condizentes com o dos autores, uma vez que não houve absorção da vibração pelos pneus na alta frequência, provavelmente isto ocorreu em função dos pneus terem água no seu interior, o que altera as propriedades de rigidez do conjunto.

No espectro de frequência de 0 a $60 \mathrm{~Hz}$ observou-se o comportamento similar dos tratamentos 
de profundidade de escarificação e velocidade de deslocamento, no entanto, para valores de 60 a $200 \mathrm{~Hz}$ verificou-se o comportamento regular das oscilações nos tratamentos (LLT-PR15-V3,5) e (LLTPR30-V3,5), porém defasados para os tratamentos LLT-PR15-V4,5 e LLT-PR30-v4,5, onde observouse irregularidade da transmissibilidade. Desta forma, constata-se a variação da velocidade em frequências acima de $60 \mathrm{~Hz}$ influenciando na forma de transmissibilidade.

Embora o lastro líquido não proporcione amplificação de vibração na baixa frequência, a vibração propagada através da estrutura do trator produziu altos valores de magnitude de vibração na interface assentooperador, se comparado com os outros tratamentos. Sendo desta forma inconveniente a utilização do lastro líquido, a qual pode produzir efeitos indesejáveis ao corpo humano, ao ser sensível às altas frequências (Figura 4).

Com a sobreposição vetorial dos tipos de lastragem, verifica-se que o lastro líquido promoveu a maior transmissibilidade de vibração ao longo do espectro, ainda que no intervalo de 0 a $20 \mathrm{~Hz}$ seus valores de transmissibilidade sejam os menores.

De maneira geral, a utilização de lastro metálico total permitiu melhores resultados de minimização de vibração para o espectro de frequência de 0 a $200 \mathrm{~Hz}$. De acordo com o modelo matemático simplificado utilizado em função do eixo logarítmico, observou-se que com a utilização de lastro metálico total, a média de transmissibilidade de 1 a $80 \mathrm{~Hz}$ correspondeu a $59 \%$ com $\mathrm{R}=0,64$; com a utilização de lastro metálico parcial a média foi de $74 \%$ com $\mathrm{R}=0,54$ e no líquido total a média foi de $88 \%$ com $\mathrm{R}=0,47$. Apesar dos valores de correlação serem baixos, permitem uma boa estimativa do comportamento da transmissibilidade de vibração no espectro de frequência.

Segundo Almeida, Tavares-Silva e Silva (2010), Smerda e Cupera (2010) e Monteiro, Lanças e Guerra (2011) a velocidade de deslocamento do trator tem influencia direta nas variáveis ergonômicas (FURLANI et al., 2007; SILVEIRA; SIERRA, 2010).

Os valores de alta rigidez e alto amortecimento promovem a minimização da vibração do motor, reduzindo assim, a transmissibilidade da vibração nas baixas frequências. Portanto, são necessárias melhorias no coxim na baixa frequência de até $20 \mathrm{~Hz}$ quando se utiliza o lastro metálico, e melhorias na eficiência sua quando usado em alta frequência, no caso de utilização do lastro líquido (Figura 5).

Figura 3 - Comportamento da transmissibilidade de vibração através do coxim no intervalo de frequência de 0 a $200 \mathrm{~Hz}$ com lastro metálico parcial

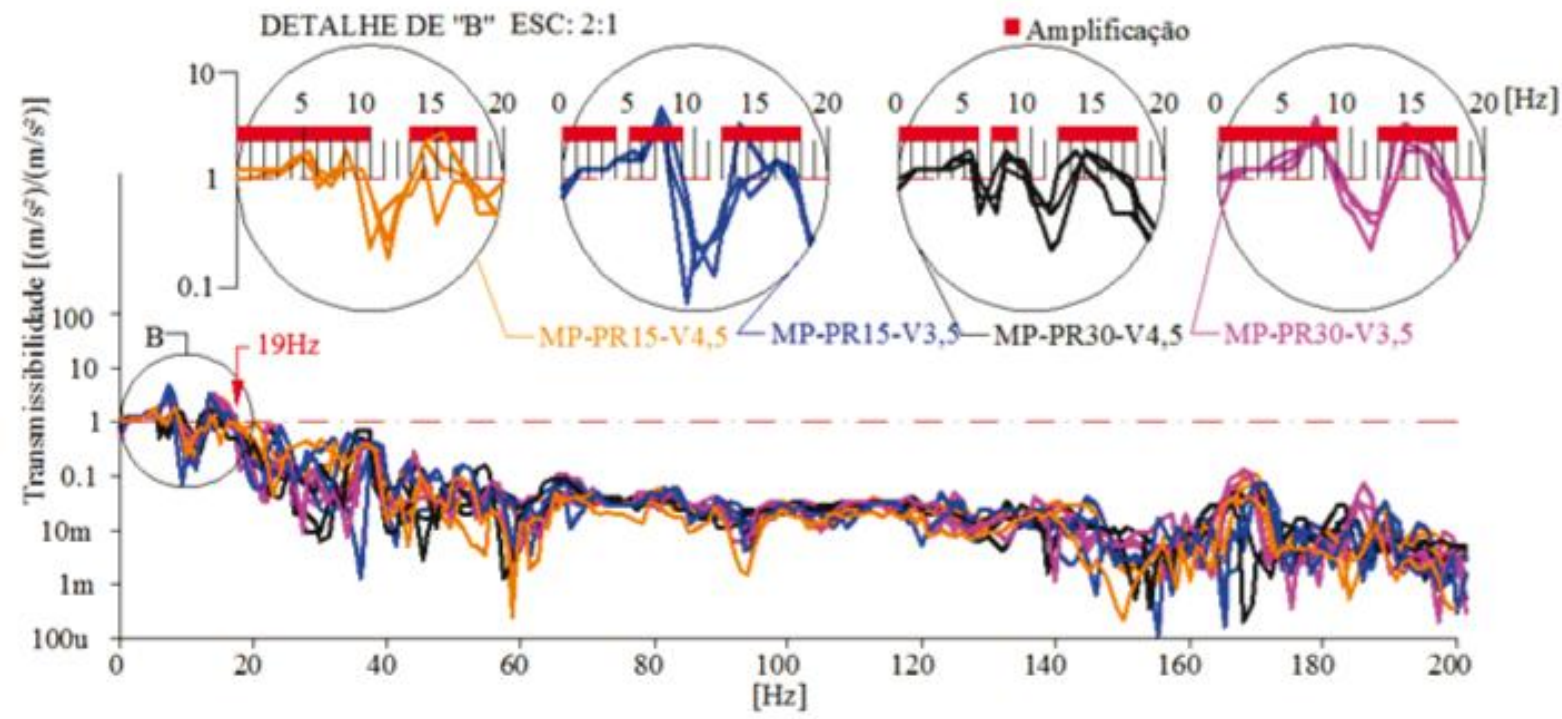

MP- metálico parcial; PR15- profundidade de escarificação de $15 \mathrm{~cm}$; PR30- profundidade de escarificação de 30 cm; V3,5- velocidade de deslocamento de $3,5 \mathrm{~km} . \mathrm{h}^{-1}$; V4,5- velocidade de deslocamento 4,5 km.h ${ }^{-1}$. Imagem gerada através do software Pulse 
Figura 4 - Comportamento da transmissibilidade de vibração através do coxim no intervalo de frequência de 0 a $200 \mathrm{~Hz}$ com lastro líquido total

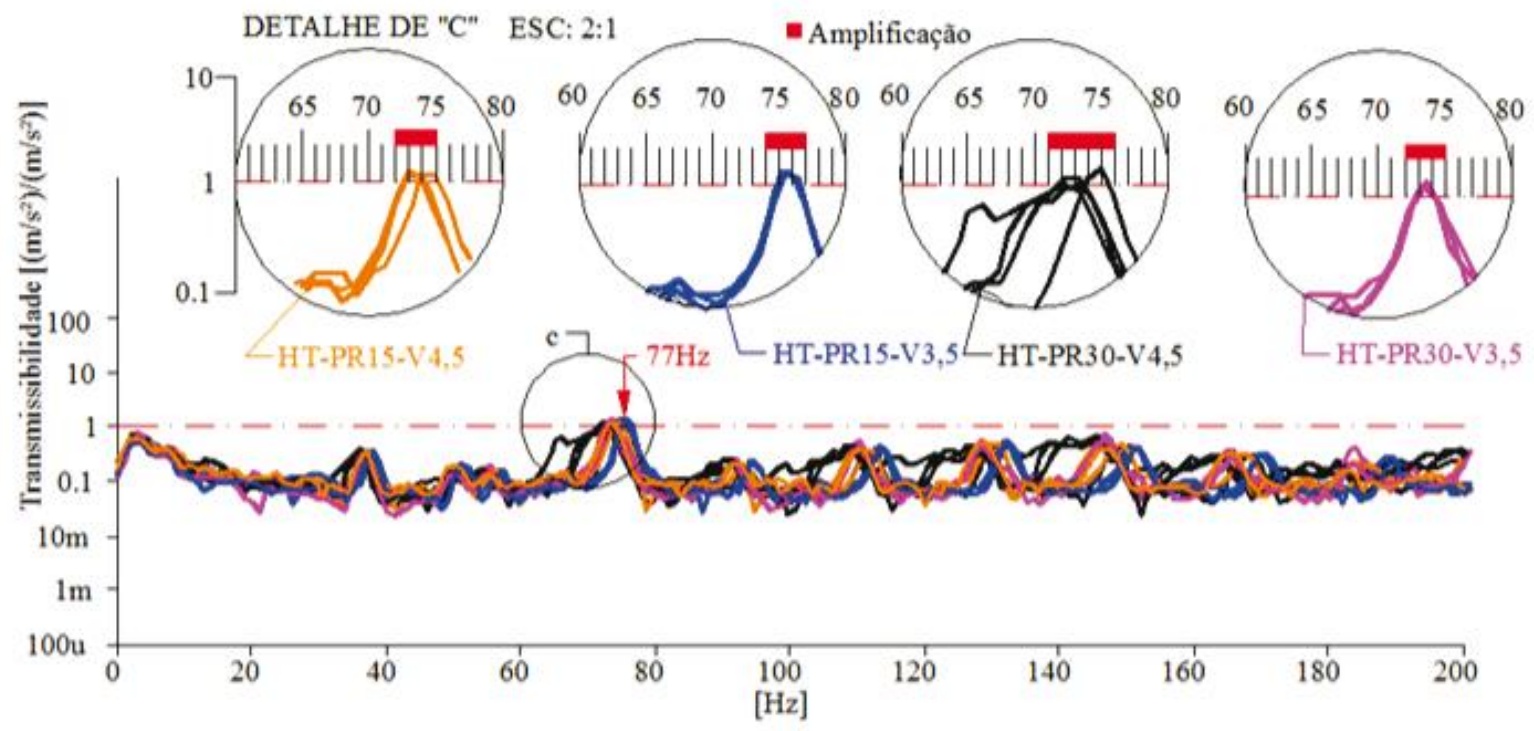

HT- liquido total; PR15- profundidade de escarificação de $15 \mathrm{~cm}$; PR30- profundidade de escarificação de $30 \mathrm{~cm}$; V3,5- velocidade de deslocamento de $3,5 \mathrm{~km} \cdot \mathrm{h}^{-1} ; \mathrm{V} 4,5$ - velocidade de deslocamento $4,5 \mathrm{~km} \cdot \mathrm{h}^{-1}$. Imagem gerada através do software Pulse

Figura 5 - Comportamento da transmissibilidade de vibração através do coxim no intervalo de frequência de 0 a $200 \mathrm{~Hz}$ com lastro metálico parcial e total e líquido total

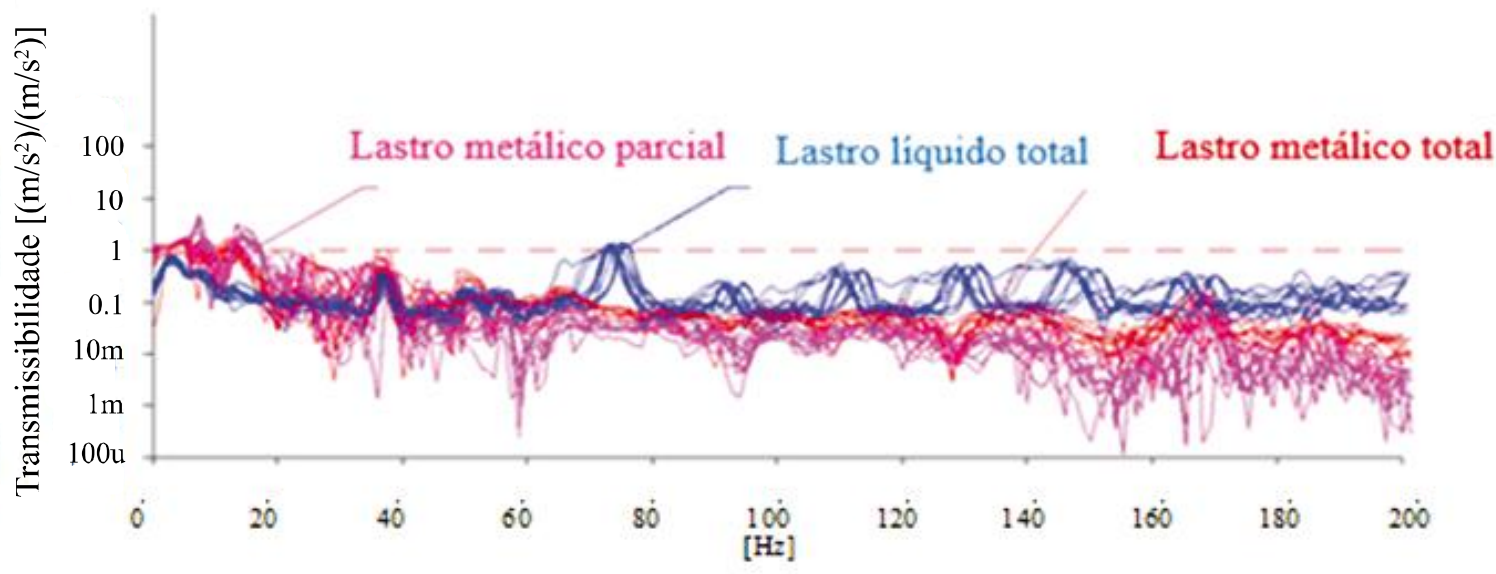

\section{CONCLUSÕES}

1.A transmissibilidade de vibração na direção vertical, através do coxim, teve amplificação no intervalo de 0 a $15 \mathrm{~Hz}$ para o lastro metálico total, de 0 a $19 \mathrm{~Hz}$ para o lastro metálico parcial e de 71 a $76 \mathrm{~Hz}$ para o lastro líquido total;

2. Os valores de transmissibilidade na análise de 1 a $80 \mathrm{~Hz}$ corresponderam a 59, 74 e $88 \%$, respectivamente para os lastros metálico total e parcial e líquido total. 


\section{REFERÊNCIAS}

ALMEIDA, R. A. S. de.; TAVARES-SILVA, C. A.; SILVA, S. de L. Desempenho energético de um conjunto trator-semeadora em função do escalonamento de marchas e rotações do motor. Revista Agrarian, v. 3, n. 7, p. 63-70, 2010.

BARBETTI, M. R. S. Estudo comparativo entre coxim hidráulico e coxim elastomérico, aplicados ao sistema de apoio do motor automotivo, 200567 f. Dissertação (Mestrado Profissionalizante em Engenharia Automotiva), Escola Politécnica da Universidade de São Paulo, São Paulo, 2005.

BARBOSA, J. A. et al. Desempenho operacional de um trator agrícola equipado alternadamente com pneus radiais e diagonais. Engenharia Agrícola, v. 25, n. 2, p. 474-480, 2005.

COELHO, H. et al. Deslizamento de rodados de tratores de pneus em diferentes operações agrícolas. Revista Ceres, v. 59, n. 3, p. 330-336, 2012.

EMPRESA BRASILEIRA DE PESQUISA AGROPECUÁRIA. Centro Nacional de Pesquisa de Solos. Sistema brasileiro de classificação de solos. Brasília: EMBRAPA, 1999. 412 p.

FRANCHINI, D. Análise do nível de vibrações verticais no assento de um trator agrícola. 2007. 138 f. Dissertação (Mestrado em Engenharia Agrícola) - Universidade Federal de Santa Maria, Santa Maria, 2007.

FREITAS, L. M. Estudo da dinâmica vertical de uma suspensão veicular do tipo MacPherson. 2006. 122 f. Dissertação (Mestrado em Engenharia Mecânica) Universidade de São Paulo, São Carlos, 2006.

FURLANI, C. E. A. et al. Desempenho operacional de semeadora-adubadora em diferentes manejos da cobertura e da velocidade. Revista Engenharia Agrícola, v. 27, n. 2, p. 456-462, 2007.

GABRIEL FILHO, A. et al. Desempenho de trator agrícola em três superfícies de solo e quatro velocidades de deslocamento. Revista Brasileira de Engenharia Agrícola e Ambiental, v. 14, n. 3, p. 333-339, 2010.

IIDA, I. Ergonomia: projeto e produção. 6. ed. São Paulo: E. Blucher, 2000. 466 p.

KUMAR, A. et al. Tractor vibration severity and driver health: a study from rural India. Journal of Agricultural Engineering Research, v. 80, n. 4, p. 313-328, 2001.

LLANILLO, R. F. et al. Evolução de propriedades físicas do solo em função dos sistemas de manejo em culturas anuais. Semina: Ciências Agrárias, v. 27, n. 2, p. 205-220, 2006.

MINETTE, L. J. et al. Avaliação dos níveis de ruído, luz e calor em máquinas de colheita florestal. Revista Brasileira de Engenharia Agrícola e Ambiental, v. 11, n. 6, p. 664667, 2007.

MONTEIRO, L. A.; LANÇAS, K. P.; GUERRA S. P. S. Desempenho de um trator agrícola equipado com pneus radiais e diagonais com três níveis de lastros líquidos. Engenharia Agrícola, v. 31, n. 3, p. 551-560, 2011.

NIETIEDT, G. H. et al. Distribuição dos comandos de operação em tratores agrícolas nacionais com até $55 \mathrm{~kW}$ de potência. Revista Brasileira de Engenharia Agrícola e Ambiental, v. 16, n. 6, p. 690-695, 2012.

OLIVEIRA, P. A. Modelagem da dinâmica vertical de coxins elastoméricos de motor através de métodos de redes neurais. 2011. 192 f. Dissertação (Mestrado em Engenharia Mecânica) - Universidade de São Paulo, São Carlos, 2011.

ROZIN, D. et al. Conformidade dos comandos de operação de tratores agrícolas nacionais com a norma NBR ISO 4253. Revista Brasileira de Engenharia Agrícola e Ambiental, v. 14, n. 9, p. 1014-1019, 2010.

RYCHTYCKYJ, N. Ergonomics analysis for vehicle assembly using artificial intelligence, Al Magazine, v. 26, n. 3, 2005, p. 41-50.

SAM, B.; KATHIRVEL, K. Vibration characteristics of walking and riding type power tillers. Biosystems Engineering, v. 95, n. 4, 517-528, 2006.

SANTOS, P. F. Avaliação dos níveis de ruído e vibração vertical no assento de um trator agrícola de pneus utilizando um sistema de aquisição automática de dados. 2002. $53 \mathrm{f}$. Dissertação (Mestrado em Engenharia Agrícola) - Universidade Federal de Viçosa, Viçosa, 2002.

SCHLOSSER, J. F. et al. Análise comparativa do peso específico dos tratores agrícolas fabricados no Brasil e seus efeitos sobre a seleção e uso. Ciência Rural, v. 35, n. 1, p. 92-97, 2005.

SERVADIO, P.; MARSILI, A.; BELFORE, N. P. Analysis of driving seat vibrations in high forward speed tractors. Biosystems Engineering, v. 97 n. 2, 171-180, 2007.

SILVA, C. B. et al. Avaliação ergonômica de uma colhedora de cana-de-açúcar. Ciência e Agrotecnologia, v. 35, n. 1, p. 179-185, 2011.

SILVEIRA, G. M.; SIERRA, J. G. Eficiência energética de tratores agrícolas fabricados no Brasil. Revista Brasileira de Engenharia Agrícola e Ambiental, v. 14, n. 4, p. 418424,2010 .

SMERDA, T.; CUPERA, J. Tire inflation and its influence on drawbar characteristics and performance - Energetic indicators of a tractor set. Journal of Terramechanics, v. 47, n. 6, p. 395- 400, 2010.

STEIN, G. J. et al. Modelling and simulation of locomotive driver's seat vertical suspension vibration isolation system. International Journal of Industrial Ergonomics, v. 38, n. 5/6, p. 384-395, 2007.

YULAN, D. Ergonomics. Beijing: Beijing institute of technology press, 2005, p. 23-25. 